\title{
Comparison of High-Resolution Computed Tomography and Real-Time Reverse Transcriptase Polymerase Chain Reaction in Diagnosis of COVID-19 Pneumonia in Intensive Care Unit Population
}

\author{
Hafiz G. Murtaza ${ }^{1}$, Nasir Javed ${ }^{1}$, Ahtesham Iqbal ${ }^{1}$, Moazma Ramzan ${ }^{1}$, Omair ul haq Lodhi ${ }^{2}$, Sadaf Majid \\ ${ }^{3}$, Kiran Abbas ${ }^{4}$, Abdul Rehman ${ }^{4}$ \\ 1. Critical Care Medicine, Shifa International Hospital Islamabad, Islamabad, PAK 2. Internal Medicine, Shifa \\ International Hospital Islamabad, Islamabad, PAK 3. Neurology, Shifa International Hospital Islamabad, Islamabad, \\ PAK 4. Medicine, Jinnah Postgraduate Medical Centre, Karachi, PAK
}

Corresponding author: Kiran Abbas, kiranabbas2020@gmail.com

\section{Abstract}

\section{Introduction}

The efficacy of high-resolution computed tomography (HRCT) chest in common respiratory infections is well-established; however, its use in the diagnosis of COVID-19 pneumonia is less popular. The previous studies have failed to establish the efficacy of HRCT in the diagnosis of COVID-19 pneumonia.

\section{Objective}

The current study aimed to assess the efficacy of HRCT as compared to a polymerase chain reaction (PCR) in diagnosing COVID-19 pneumonia in patients in our setting.

\section{Methodology}

A prospective observational study was conducted at the Department of Chest Medicine, Shifa International Hospital from April 2020 to December 2020. A total of 250 patients were admitted to medical intensive care units. Findings of HRCT and PCR were documented. The accuracy of HRCT compared with PCR was assessed. Data were analyzed using SPSS version 24 (IBM Corp., Armonk, NY).

\section{Results}

COVID-19 infection was more prevalent in male patients (62.8\% vs $37.2 \%)$. The mean age was 60 years (interquartile range, IQR, 49-72). Sensitivity and specificity of HRCT segregated into typical, indeterminate, and atypical HRCT were $(94.8 \%, 56.8 \%),(92.7 \%, 47.2 \%)$, and (91.7\%, 76.8\%), respectively. The positive

Review began 02/18/2021 Review ended 02/19/2021 Published 02/28/2021

\section{() Copyright 2021}

Murtaza et al. This is an open access article distributed under the terms of the Creative Commons Attribution License CC-BY 4.0., which permits unrestricted use, distribution, and reproduction in any medium, provided the original author and source are credited. predictive value for typical HRCT was $84.3 \%$ ( $\mathrm{p} \leqslant 0.001)$.

\section{Conclusion}

We concluded that typical HRCT findings have diagnostic utility in the diagnosis of COVID pneumonia. Similarly, a negative HRCT chest reliably excludes the possibility of COVID pneumonia. HRCT chest is a reliable alternative to RT-PCR.

Categories: Radiology, Infectious Disease, Pulmonology

Keywords: polymerase chain reactions, diagnostic imaging, computed tomography, $\mathrm{x}$-ray

\section{Introduction}

By the end of 2019, a unique viral infection started to spread all across the globe which was initially reported in Wuhan, China [1]. It was suspected to have spread from phinolphus bats to humans and was regarded as SARS-CoV-2 or COVID-19 infection [2]. As of now, COVID-19 has infected 89.7 million people all around the world with 1.93 million deaths. In Pakistan, 502,000 cases have been reported with 10,644 deaths, nationally $[3,4]$.

The COVID-19 infection is mainly spread from human to human via respiratory droplets during sneezing and coughing. The average incubation period of this virus is between 2.1 days and 11.1 days during which the infected person remains asymptomatic. This greatly contributes to the transmission of the virus [5].

Currently, research is focused on developing an effective vaccine against the virus, however, the results have 
been inconsistent. Therefore, it is of extreme importance to detect the infected patients and minimize their exposure to the healthy population. Immediate isolation of patients infected with the COVID-19 virus is the most effective way to stop the spread to a further population $[6,7]$.

Currently, the COVID-19 infection is diagnosed with the aid of detection of viral nucleic acids using realtime polymerase chain reaction (RT-PCR) or gene sequencing for respiratory or blood specimens [5]. However, there are some challenges associated with these diagnostic modalities. For instance, sample collection and transportation is restricted by limited workforce and unavailability of PCR kit. The total positive rate of RT-PCR for COVID-19 throat swab cultures is reported to be somewhere between $30 \%$ and $60 \%[6]$.

On the other hand, a recent study by Qureshi et al. reported that high-resolution computed tomography (HRCT) thorax is a substantially useful modality in establishing the diagnosis of COVID-19 pneumonia [8]. Furthermore, HRCT is less time-consuming and may reveal abnormalities in the lung parenchyma consistent with features of COVID-19 pneumonia in individuals with negative PCR of nasal or throat swab culture results [7]. The comparative studies between HRCT chest and RT-PCR in the ICU population have not been carried out so far. That is why we are conducting this study to analyze the diagnostic value of HRCT chest as compared to RT-PCR in the ICU population and also to determine the diagnostic utility of HRCT chest in comparison to RT-PCR in the diagnosis of COVID pneumonia in the ICU population.

\section{Materials And Methods}

An observational prospective research was undertaken at the Intensive care units, Shifa International Hospital, Islamabad, Pakistan between April 2020 to December 2020 for a duration of nine months. The study was first approved by the ethical committee of Shifa International hospital with IRB reference number 128-948-2020. Informed verbal consent was taken from all patients. The study included all patients presenting with signs and symptoms of COVID-19 infection including cough, shortness of breath, fever, and chest pain, admitted to the Intensive care units of the hospital. A non-probability consecutive sampling technique was applied.

Sociodemographic variables and clinical history of patients were obtained from the guardians in detail and recorded in a predefined proforma. The variables recorded included, gender, age, marital status, presenting complaints, duration of symptoms, and comorbidities. Only patients with HRCT chest and RT-PCR test on nasopharyngeal swab findings of no more than two days old since the admission to ICU were included in the study. The sample size was calculated using an electronic calculator. A total of 250 patients fulfilled the eligibility criteria for participation in the study.

A patient was considered as a "confirmed" case of COVID-19 pneumonia if a single positive RT-PCR result was positive. A patient with two consecutive negative RT-PCR results two days apart were considered as "negative" cases. Patients were tested for COVID-19 pneumonia using real-time reverse transcriptasepolymerase chain reaction (RT-PCR) tests to detect viral nucleic acid and HRCT Chest.

All HRCT chests were performed in the radiology department by an experienced radiologist. Patients were asked to lay in the supine position with a tube voltage of $120 \mathrm{kV}$ and slice thickness of $1 \mathrm{~cm}[8]$.

HRCT findings were categorized into four groups, i.e., (1) typical, (2) indeterminate, (3) atypical, and (4) negative for COVID-19. The main findings of HRCT in patients with COVID-19 pneumonia were defined as the presence of bilateral peripheral multilobar or multifocal ground-glass opacities (GGO) with or without consolidation with interseptal thickening (IST) or peripheral bilateral GGO with or without consolidation with IST or typical crazy paving pattern or reverse halo sign or other findings of organizing pneumonia.

HRCT intermediate for COVID-19 was defined as the presence of bilateral GGO and consolidation or multifocal, perihilar, or unilateral GGO with or without peripheral consolidation without any particular distribution or a few very small non-rounded or non-peripheral GGO. An atypical COVID-19 picture was characterized by consolidation in the isolated lobe without any GGO, small discrete nodules, cavitation, thickening of the septa, or lung effusion.

HRCT chest was taken as an index test because, in comparison to RRT-PCR and immunological testing, it is non-invasive, readily available, easy to perform, replicable, and does not depend on the duration of illness. Its reported sensitivity and specificity are $91.9 \%$ and $25.1 \%$, respectively [8].

Data analysis was done by using statistical software for social sciences (SPSS version 24, IBM Corp., Armonk, $\mathrm{NY}$ ). We presented categorical data in frequencies and percentages and age in median and interquartile range (IQR) being non-normal distribution. Considering the RT-PCR test as a reference, we calculated sensitivity, specificity, positive predictive values using contingency tables with $95 \%$ confidence intervals. Statistical significance was considered at a p-value of $<0.05$. 


\section{Cureus}

\section{Results}

Most of our study population consisted of males 157 (62.8\%) as compared to females 93 (37.2\%). The mean age was 60 years (IQR 49-72). We divided our population into two age groups; $\geqslant 60$ years and $<60$ years. Amongst males, most of the patients were $\geqslant 60$ years ( $54.8 \mathrm{vs} 45.2$ ) whereas in the female group most of the patients were $<60$ years ( 51.6 vs 48.4 ). Negative PCR results were $50.8 \%$ in $<60$ years patients and $55.6 \%$ in $\geqslant 60$ years age group; however, it was not statistically significant $(\mathrm{p}=0.375$; Figure 1$)$.

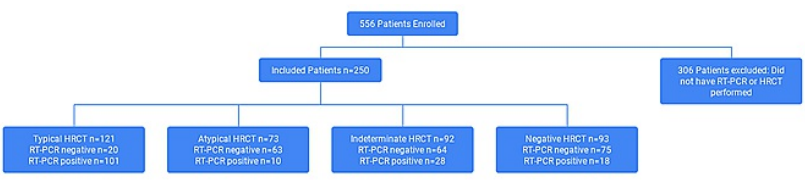

\section{FIGURE 1: Flow chart of study participants}

HRCT: high-resolution computed tomography, RT-PCR: real-time polymerase chain reaction.

This study reported that the sensitivity and specificity of typical and atypical HRCT in comparison with RRTPCR results were $91 \%$ and $76 \%$, respectively. The positive predictive value was $83.4 \%$ versus the negative predictive value which was $86.3 \%$, p-value $<0.005$ (Table 1 ).

\begin{tabular}{|c|c|c|c|}
\hline Test Outcome & PCR Positive & PCR Negative & \\
\hline HRCT typical & 101 & 20 & Positive predictive value $=83.4 \%$ \\
\hline \multirow[t]{2}{*}{ HRCT atypical } & 10 & 63 & Negative predictive value $=86.3 \%$ \\
\hline & Sensitivity=91\% & Specificity=76\% & p-Value $<0.005$ \\
\hline
\end{tabular}

TABLE 1: Sensitivity and specificity of typical and atypical HRCT in comparison with RT-PCR outcomes

HRCT: high-resolution computed tomography, RT-PCR: real-time polymerase chain reaction.

The sensitivity and specificity of typical and indeterminate HRCT in comparison with RRT-PCR results were $78.2 \%$ and $76.1 \%$, respectively. The positive predictive was $83.4 \%$ versus the negative predictive value which was 69.6\%, p-value $<0.005$ (Table 2).

\begin{tabular}{|c|c|c|c|}
\hline Test Outcome & PCR Positive & PCR Negative & \\
\hline HRCT typical & 101 & 20 & Positive predictive value= $83.4 \%$ \\
\hline \multirow[t]{2}{*}{ HRCT indeterminate } & 28 & 64 & Negative predictive value $=69.6 \%$ \\
\hline & Sensitivity=78.2\% & Specificity=76.1\% & p-Value $<0.005$ \\
\hline
\end{tabular}

TABLE 2: Sensitivity and specificity of typical and Indeterminate HRCT in comparison with RRTPCR outcomes

HRCT: high-resolution computed tomography, RRT-PCR: real-time reverse transcriptase-polymerase chain reaction.

The sensitivity and specificity of typical and negative HRCT in comparison with RRT-PCR results were $84.5 \%$ and $78.9 \%$, respectively. The positive predictive was $83.4 \%$ versus the negative predictive value which was $80.6 \%$, p-value $<0.005$ (Table 3). 


\section{Cureus}

\begin{tabular}{|c|c|c|c|}
\hline Test Outcome & PCR Positive & PCR Negative & \\
\hline HRCT typical & 101 & 20 & Positive predictive value $=83.4 \%$ \\
\hline \multirow[t]{2}{*}{ HRCT negative } & 18 & 75 & Negative predictive value $=80.6 \%$ \\
\hline & Sensitivity $=84.5 \%$ & Specificity $=78.9 \%$ & $\mathrm{p}$-Value $<0.005$ \\
\hline \multicolumn{4}{|c|}{$\begin{array}{l}\text { TABLE 3: Sensitivity and specificity of typical and negative HRCT in comparison with RRT-PCF } \\
\text { outcomes }\end{array}$} \\
\hline \multicolumn{4}{|c|}{ HRCT: high-resolution computed tomography, RRT-PCR: real-time reverse transcriptase-polymerase chain reaction. } \\
\hline
\end{tabular}

\section{Discussion}

The current study evaluated the prospects of HRCT being used as a diagnostic modality in patients suspected of COVID-19 infection. Due to the reduced sensitivity of RT PCR, many patients remain unidentified. This delays treatment and also contributes to the transmission of COVID-19 infection [8-10]. Computed tomography is technically not as demanding as RRT-PCR. It is routinely used in the diagnosis of pneumonia. It also provides results faster as compared to COVID-19 PCR [11-13].

A typical HRCT picture of COVID-19 pneumonia as reported by recent studies shows GGO with concomitant multifocal patchy consolidates or interstitial or parenchymal changes [7]. Similar findings in HRCT of patients with negative RRT-PCR reports were observed indicating that HRCT can diagnose the abnormalities in the lung of patients infected with COVID-19 even when PCR is false negative [12]. A meta-analysis has concluded the sensitivity of RT-PCR on the nasopharyngeal swab and HRCT chest $68.1 \%$ to $78 \%$ and $91.9 \%$, respectively, but, the accuracy of RT-PCR depends on the adequacy of sample and duration of illness and takes hours in reporting $[9,10]$.

In our study, males were more commonly affected as compared to females (62.8\% vs $37.2 \%)$. COVID-19 is common in males as they have more expression of receptors for coronavirus attachment (ACE 2), higher chances of smoking and alcoholism. Females are more resilient to COVID-19 infection and responsible behavior to COVID-19 pandemic as compared to males [14]. Typical HRCT findings had been more popular in our study population and had a statistically significant association with PCR results. The sensitivity of typical HRCT was $91.7-94.8 \%$ which is comparable to the results of a meta-analysis of 16 studies that showed that the HRCT chest is $86-96 \%$ sensitive in the diagnosis of COVID-19 pneumonia. This replication of results is due to the fact that bilateral GGOs and multilobe consolidations were typical findings on the HRCT chest and we adopted the same pattern to define a typical HRCT chest [15]. Salehi et al. have also described the same findings in their systematic review to define typical findings on HRCT [16]. Our results showed quite a higher $56.8 \%$ specificity of HRCT chest which is higher than $33 \%$ reported by Xu et al. in their meta-analysis [15]. This difference may be due to superimposed viral pneumonia which can reduce specificity of HRCT chest, heterogeneity of expertise of radiologists, and severity of illness in the COVID-19 population.

In a local study by Qureshi et al., HRCT reported sensitivity and specificity of $97.41 \%$ and $80 \%$, respectively, with a positive predictive value of $99.12 \%$ and a negative predictive value of $57.14 \%$. Furthermore, they reported the diagnostic accuracy of HRCT for COVID-19 to be $96.69 \%$ [8].

This concludes that in patients with suspected COVID-19 pneumonia, the HRCT chest in addition to RRTPCR can be used for the diagnosis of COVID-19 pneumonia. Long et al., reveals that the sensitivity of CT scan was $97.2 \%$, whereas the PCR sensitivity was $83.3 \%$. The study authors recommend that it is better to isolate patients with typical findings of COVID-19 on chest CT even when PCR is negative for COVID-19 nucleic acids [13].

\section{Conclusions}

In conclusion, typical HRCT findings have diagnostic utility in the diagnosis of COVID-19 pneumonia. Similarly, negative HRCT chest reliably excludes the possibility of COVID-19 pneumonia. Although RT-PCR is a gold standard test to diagnose COVID-19, HRCT can be useful in those circumstances where RT-PCR is awaited and isolation of COVID-19 cases is a concern. Sometimes, there is high clinical suspicion of COVID19 but RT-PCR may be falsely negative. Moreover, resources for RT-PCR may run short in view of the increasing number of cases at some centers. In such circumstances, HRCT findings can be a possible alternative. 


\section{Additional Information \\ Disclosures}

Human subjects: Consent was obtained or waived by all participants in this study. Shifa International Hospitals, Ltd. issued approval IRB\#128-948-2020. Animal subjects: All authors have confirmed that this study did not involve animal subjects or tissue. Conflicts of interest: In compliance with the ICMJE uniform disclosure form, all authors declare the following: Payment/services info: All authors have declared that no financial support was received from any organization for the submitted work. Financial relationships: All authors have declared that they have no financial relationships at present or within the previous three years with any organizations that might have an interest in the submitted work. Other relationships: All authors have declared that there are no other relationships or activities that could appear to have influenced the submitted work.

\section{References}

1. Huang C, Wang Y, Li X, et al.: Clinical features of patients infected with 2019 novel coronavirus in Wuhan, China. Lancet. 2020, 395:497-506. 10.1016/S0140-6736(20)30183-5

2. Clinical management of severe acute respiratory infection when novel coronavirus ( $\mathrm{nCoV}$ ) infection is suspected: interim guidance. (2020). Accessed: January 25, 2020: https://apps.who.int/iris/handle/10665/330854.

3. Dong L, Hu S, Gao J: Discovering drugs to treat coronavirus disease 2019 (COVID-19). Drug Discov Ther. 2020, 14:58-60. 10.5582/ddt.2020.01012

4. Epidemiology Working Group for NCIP Epidemic Response, Chinese Center for Disease Control and Prevention: The epidemiological characteristics of an outbreak of 2019 novel coronavirus diseases (COVID19)-in China. Zhonghua Liu Xing Bing Xue Za Zhi. 2020, 41:113-122. 10.3760/cma.j.issn.02546450.2020.02.003

5. Jin Y-H, Cai L, Cheng Z-S, et al.: A rapid advice guideline for the diagnosis and treatment of 2019 novel coronavirus (2019-nCoV) infected pneumonia (standard version). Mil Med Res. 2020, 7:4. 10.1186/s40779020-0233-6

6. Evaluating the accuracy of different respiratory specimens in the laboratory diagnosis and monitoring the viral shedding of 2019-nCoV infections. (2020). Accessed: February 26, 2021: http://medrxiv.org/content/10.1101/2020.02.11.20021493v2.article-metrics.

7. Chung M, Bernheim A, Mei X, et al.: CT imaging features of 2019 novel coronavirus (2019-nCoV) . Radiology. 2020, 295:202-227. 10.1148/radiol.2020200230

8. Qureshi AR, Akhtar Z, Irfan M, Sajid M, Ashraf Z: Diagnostic value of HRCT-Thorax for pandemic COVID-19 pneumonia in Pakistan. J Rawalpindi Med College. 2020, 13:77-84. 10.37939/jrmc.v24iSupp-1.1443

9. Böger B, Fachi MM, Vilhena RO, de Fátima Cobre A, Tonin FS, Pontarolo R: Systematic review with metaanalysis of the accuracy of diagnostic tests for COVID-19. Am J Infect Control. 2020, 49:21-29. 10.1016/j.ajic.2020.07.011

10. Watson J, Whiting PF, Brush JE: Interpreting a COVID-19 test result. BMJ. 2020, 369:m1808. 10.1136/bmj.m1808

11. Huybens EM, Bus MP, Massaad RA, et al.: What is the preferred screening tool for COVID-19 in asymptomatic patients undergoing a surgical or diagnostic procedure?. World J Surg. 2020, 44:3199-3206. 10.1007/s00268-020-05722-9

12. Huybens EM, Bus MP, Massaad RA, et al.: Screening with HRCT chest and PCR testing for COVID-19 in asymptomatic patients undergoing a surgical or diagnostic procedure. Br J Surg. 2020, 107:e384-e385. 10.1002/bjs.11822

13. Long C, Xu H, Shen Q, et al.: Diagnosis of the Coronavirus disease (COVID- 19): rRT-PCR or CT? . Eur J Radiol. 2020, 25:108961. 10.1016/j.ejrad.2020.108961

14. Bwire GM: Coronavirus: why men are more vulnerable to COVID-19 than women? [PREPRINT] . SN Compr Clin Med. 2020, 1-4. 10.1007/s42399-020-00341-w

15. Xu B, Xing Y, Peng J, et al.: Chest CT for detecting COVID- 19: a systematic review and meta-analysis of diagnostic accuracy. Eur Radiol. 2020, 30:5720-5727. 10.1007/s00330-020-06934-2

16. Salehi S, Abedi A, Balakrishnan S, Gholamrezanezhad A: Coronavirus disease 2019 (COVID- 19): a systematic review of imaging findings in 919 patients. Am J Roentgenol. 2020, 215:87-93. 10.2214/AJR.20.23034 